\title{
Early Improvement with Vortioxetine Predicts Response and Remission: A Post Hoc Analysis of Data from a Clinical Trial Conducted in Japan
}

\author{
Takeshi Inoue (D) \\ Shinji Fujimoto $\mathbb{D}^{2}$ \\ Tatsuro Marumoto ${ }^{2}$ \\ Tadayuki Kitagawa ${ }^{3}$ \\ Kazuyuki Ishida ${ }^{3}$ \\ Tadashi Nakajima ${ }^{2}$ \\ Yoshiya Moriguchi $\mathbb{D}^{4}$ \\ Keita Fujikawa ${ }^{2}$ \\ Koichiro Watanabe ${ }^{5}$ \\ 'Department of Psychiatry, Tokyo \\ Medical University, Tokyo, Japan; ${ }^{2} J a p a n$ \\ Medical Office, Takeda Pharmaceutical \\ Co., Ltd, Tokyo, Japan; ${ }^{3}$ Takeda \\ Development Center - Japan, Takeda \\ Pharmaceutical Co., Ltd, Osaka, Japan; \\ ${ }^{4}$ Medical Affairs, Lundbeck Japan K.K., \\ Tokyo, Japan; ${ }^{5}$ Department of \\ Neuropsychiatry, Kyorin University \\ School of Medicine, Tokyo, Japan
}

Correspondence: Takeshi Inoue Department of Psychiatry, Tokyo Medical University, 6-7-I Nishishinjuku, Shinjuku-ku, Tokyo, 160-0023, Japan

Tel +8I-3-3342-6I II (ext. 5754)

Fax +8I-3-3340-4499

Email tinoue@tokyo-med.ac.jp

\begin{abstract}
Aim: Several weeks of treatment with an antidepressive agent may be required before efficacy is demonstrated in patients with major depressive disorder. This study investigated the predictive value of early partial improvement with vortioxetine for treatment response and remission.

Methods: This was a post hoc analysis of an 8-week, randomized, double-blind, placebocontrolled, Phase 3 study of vortioxetine (10 mg or $20 \mathrm{mg}$ ) in Japanese patients aged 20-75 years with recurrent major depressive disorder and a Montgomery-Åsberg Depression Rating Scale (MADRS) score of at least 26. The key outcomes were the predictive value of early partial improvement (reduction in MADRS total score of $\geq 20 \%$ from baseline to week 2 ) with vortioxetine for MADRS response ( $\geq 50 \%$ decrease in score from baseline) and remission (decrease in score to $\leq 10$ ) at week 8 .
\end{abstract}

Results: Relevant data were available for 478 patients; $62 / 158$ patients receiving placebo, 71/162 receiving vortioxetine $10 \mathrm{mg}$, and 66/158 receiving vortioxetine $20 \mathrm{mg}$ were early improvers. Early improvers receiving vortioxetine (10 $\mathrm{mg}$ or $20 \mathrm{mg}$ ) were more likely than non-early improvers to achieve a week 8 response $(71.2-73.2 \%$ vs $29.7-38.0 \%)$ or remission $(50.7-51.5 \%$ vs $17.4-18.7 \%$ ). Positive predictive values for response and remission with vortioxetine were $\sim 70 \%$ and $\sim 50 \%$, respectively; negative predictive values were $\sim 70 \%$ and $\sim 80 \%$, respectively.

Conclusion: Improvement with vortioxetine may be predicted by early partial improvement in MADRS score. Some patients may benefit from longer-term treatment even without early improvement, another finding that may aid clinical decision-making. ClinicalTrials.gov registration for primary study: NCT02389816.

Keywords: antidepressant, Japanese, major depressive disorder, vortioxetine

\section{Introduction}

Major depressive disorder (MDD) is a common mental disorder that burdens not only the affected individuals, but also their community and the public health system and economy of their country in terms of substantial direct medical costs and indirect social costs. ${ }^{1,2}$ Although numerous medications are available for the management of MDD, several weeks of treatment may be required to determine the effectiveness of most antidepressants. ${ }^{3}$ The precise mechanism leading to this delayed treatment effect remains to be elucidated; however, biophysiological changes in the brain, such as the downregulation of monoamine receptors and reconstruction of the neural network, are thought to be involved. ${ }^{3}$

It is indisputable that early achievement of treatment response or remission are the most favorable outcomes for patients with MDD. ${ }^{3,4}$ When no satisfactory 
response is obtained in a patient receiving antidepressant therapy, physicians are typically advised to consider alternatives to the patient's treatment regimen to obviate unnecessary suffering and exposure to a high risk of suicide. ${ }^{5,6}$ The Japanese Society of Mood Disorders recommends that patients be switched to an alternative antidepressant when they do not respond to "a sufficient dose for a sufficient period of time", ${ }^{7}$ which can help to avoid premature termination of an antidepressant and thereby minimize the risk of denying patients the long-term potential of that treatment. The risk of premature termination may be further minimized by predicting future treatment response; a growing body of evidence suggests that the potential efficacy of an antidepressive agent may be predicted by an early change in rating scales for depression. ${ }^{4}$ A systematic review of 17 randomized controlled trials enrolling more than 14,000 patients with MDD revealed that early improvement could predict response and remission after 5-12 weeks of treatment with high sensitivity and low-to-moderate specificity. ${ }^{8}$ Similarly, a pooled analysis demonstrated that inadequate long-term treatment outcomes may be predicted by insufficient improvements during the early phase of treatment, ${ }^{9}$ providing further evidence for the predictive value of early treatment response.

Vortioxetine is a multimodal antidepressant with a unique mechanism of action. As a serotonin reuptake inhibitor and modulator (S-RIM), vortioxetine not only inhibits serotonin reuptake in the synaptic cleft but also directly modulates serotonin release via several 5-HT receptors. ${ }^{10}$ Vortioxetine (at daily doses of $10 \mathrm{mg}$ and $20 \mathrm{mg}$ ) has previously demonstrated significant improvement of MDD symptoms compared with placebo in eleven 6-8-week clinical trials. ${ }^{11}$ In a more recent phase 3 randomized controlled trial enrolling Japanese patients with MDD (CCT-004), a significant improvement from baseline in the Montgomery-Åsberg Depression Rating Scale (MADRS) total score was demonstrated at weeks 6 and 8 with vortioxetine $20 \mathrm{mg}$ and at week 8 with vortioxetine $10 \mathrm{mg}$, each compared with placebo $\left(P<0.05\right.$ for both doses). ${ }^{12}$

The robustness of data collected from the phase 3 trial was the basis for several post hoc analyses, including that discussed here. In other analyses, the authors investigated the therapeutic potential of vortioxetine for anxious depression and for anhedonia symptoms in depression in Japanese patients, the results of which have been published in this journal.
Herein we describe a post hoc analysis investigating the predictive value of early improvement in the depression scale with vortioxetine for future efficacy in Japanese patients with MDD.

\section{Patients and Methods Study Design}

This was a post hoc analysis of CCT-004, a multicenter, randomized, double-blind, placebo-controlled, parallelgroup, phase 3 trial investigating the safety and efficacy of vortioxetine in Japanese patients with MDD (ClinicalTrials. gov identifier: NCT02389816). ${ }^{12}$ The trial was conducted from April 10, 2015 to 16 March 16, 2018. Eligible patients completed a $1-3-$ week screening period and a single-blind 1-week placebo run-in period to minimize the placebo response. Patients were then randomly assigned (1:1:1) to receive placebo, vortioxetine $10 \mathrm{mg}$, or vortioxetine $20 \mathrm{mg}$, once daily for 8 weeks during a double-blind treatment period. Two vortioxetine doses were investigated because both $10 \mathrm{mg}$ and $20 \mathrm{mg}$ demonstrated nominally significant improvements in MADRS total score and response rate, and acceptable tolerability, in the secondary analysis of a randomized, placebo-controlled, multinational, 8-week, Phase 2/3 trial of vortioxetine that included Japanese participants with MDD. ${ }^{13}$

After the treatment period, study drugs were withdrawn, at which point patients entered a 4-week safety follow-up period for the analysis of outcomes of adverse events. All patients provided written informed consent before study entry. Investigations were conducted in accordance with the Declaration of Helsinki and the International Council on Harmonisation tripartite guideline on Good Clinical Practice. ${ }^{14}$ The clinical study protocol, the investigator's brochure, a sample informed consent form, and other study related documents were reviewed and approved by the local or central Institutional Review Boards of all study sites (Supplemental Information S1).

\section{Patients}

Patients included in this post hoc analysis were those in the full analysis set of the primary study for whom week 2 MADRS total score data were available. Full inclusion, exclusion, and study entry criteria have been described previously. ${ }^{12}$ Briefly, patients were adults aged 20-75 years and had a primary diagnosis of recurrent MDD according to the Diagnostic and Statistical Manual of Mental Disorders (4th edition, text revision; DSM-IV-TR) criteria for $296.3 \mathrm{x},{ }^{15}$ corresponding to F33 
in ICD-10, ${ }^{16}$ with the current major depressive episode having lasted 3-12 months (inclusive) at the time of enrollment for clear diagnostic certainty and exclusion of possible concurrent mental disorders. Patients were required to have a MADRS total score $\geq 26$ from screening to study drug initiation, to include patients with moderate or severe MDD. Patients were excluded if they had previously not responded to two or more antidepressants prescribed for at least 6 weeks, to include suitable subjects for the evaluation of drug effect, or excluded if they tested positive in a urine drug-screening test. Patients who were diagnosed with any psychiatric disorder as defined by the DSM-IV-TR and patients who had any substance-induced mood disorder (excluding nicotine- and caffeine-related disorders) were also excluded.

\section{Efficacy Outcomes}

The key outcomes of this analysis were the predictive value of early improvement (reduction in MADRS total score of at least $20 \%$ from baseline to week 2) ${ }^{17}$ for MADRS response and remission at week 8. Patients who did not satisfy the criteria for early improvement were defined as non-early improvers. MADRS response was defined as a decrease of at least 50\% in MADRS total score from baseline, and remission was defined as a decrease in MADRS total score to 10 or lower.

\section{Statistical Analyses}

Patient demographics and baseline characteristics were summarized descriptively. MADRS response rates and remission rates at week 8 (last observation carried forward) were calculated for early improvers and non-early improvers in each treatment group (placebo, vortioxetine $10 \mathrm{mg}$, and vortioxetine $20 \mathrm{mg}$ ). Sensitivity and specificity of early improvement for prediction of future response and remission were calculated as follows: sensitivity $=$ [early improvers who became responders or remitters $\div$ (early improvers who became responders or remitters + non-early improvers who became responders or remitters) $] \times 100$; specificity $=$ [non-early improvers who did not become responders or remitters $\div$ (non-early improvers who did not become responders or remitters + early improvers who did not become responders or remitters) $] \times 100$. Positive and negative predictive values were calculated as follows: positive predictive value $=$ (early improvers who became responders or remitters $\div$ all early improvers $) \times 100$; negative predictive value $=$ (non-early improvers who did not become responders or remitters $\div$ all non-early improvers $) \times 100$.

\section{Results}

\section{Patients}

Patient demographics and baseline characteristics, including disease severity (MADRS score), were well balanced between treatment groups in the primary study and have been described previously. ${ }^{12}$ Of the 493 patients randomly assigned to treatment, 478 were included in this post hoc analysis; of these, 158 received placebo, 162 received vortioxetine $10 \mathrm{mg}$, and 158 received vortioxetine $20 \mathrm{mg}$.

\section{Study Assessments}

In this post hoc analysis, 62 patients $(39.2 \%)$ receiving placebo, 71 patients $(43.8 \%)$ receiving vortioxetine $10 \mathrm{mg}$, and 66 patients $(41.8 \%)$ receiving vortioxetine $20 \mathrm{mg}$ met the criteria for early improvement. Compared with non-early improvers, early improvers in the vortioxetine $10 \mathrm{mg}$ and $20 \mathrm{mg}$ groups were observed to be more likely to achieve a week 8 response (10 mg: $29.7 \%$ vs $73.2 \%$, $20 \mathrm{mg}$ : $38.0 \%$ vs $71.2 \%$, respectively) or remission (10 mg: $18.7 \%$ vs $50.7 \%$, $20 \mathrm{mg}$ : $17.4 \%$ vs $51.5 \%$, respectively). A similar trend was seen for patients receiving placebo. Among non-early improvers, the future response and remission rate of patients receiving vortioxetine $20 \mathrm{mg}$ was approximately twice that of patients receiving placebo (Table 1).

The sensitivity of early improvement for the prediction of future response and remission was moderate (approximately 60-80\%) (Table 2). With respect to positive predictive values, an average of $72.3 \%$ of the early improvers receiving vortioxetine (10 $\mathrm{mg}$ or $20 \mathrm{mg}$ ) achieved a response at week 8 and $51.1 \%$ achieved remission, as indicated earlier. Positive predictive values for response and remission with placebo were $62.9 \%$ and $43.5 \%$, respectively. An assessment of negative predictive values for patients who received vortioxetine (10 mg or $20 \mathrm{mg}$ ) who were non-early improvers showed that $66.1 \%$ did not achieve a response at week 8 and $82.0 \%$ did not achieve remission. Patients receiving vortioxetine $20 \mathrm{mg}$ had lower negative predictive values for MADRS response than those receiving vortioxetine $10 \mathrm{mg}$; values for remission were similar for the vortioxetine groups (approximately $82.0 \%$ ). Negative predictive values for response and remission with placebo were $79.2 \%$ and $92.7 \%$, respectively (Table 2).

\section{Discussion}

It is well recognized that several weeks of treatment are required for most antidepressants to demonstrate their effectiveness. ${ }^{3}$ Additionally, the heterogeneous nature of 
Table I Proportion of Patients Who Achieved Early Improvement and/Or Week 8 Response or Remission

\begin{tabular}{|l|l|l|l|l|l|l|}
\hline \multirow{2}{*}{} & \multicolumn{2}{|l|}{ Placebo (n= 158) } & \multicolumn{2}{l|}{ Vortioxetine I0 mg (n=162) } & \multicolumn{2}{l|}{ Vortioxetine 20 mg (n=158) } \\
\cline { 2 - 7 } & $\begin{array}{l}\text { Early } \\
\text { Improver }\end{array}$ & $\begin{array}{l}\text { Non-Early } \\
\text { Improver }\end{array}$ & $\begin{array}{l}\text { Early } \\
\text { Improver }\end{array}$ & $\begin{array}{l}\text { Non-Early } \\
\text { Improver }\end{array}$ & $\begin{array}{l}\text { Early } \\
\text { Improver }\end{array}$ & $\begin{array}{l}\text { Non-Early } \\
\text { Improver }\end{array}$ \\
\hline MADRS responder, $\mathrm{n}(\%)$ & $39(62.9)$ & $20(20.8)$ & $52(73.2)$ & $27(29.7)$ & $47(71.2)$ & $35(38.0)$ \\
MADRS non-responder, $\mathrm{n}(\%)$ & $23(37.1)$ & $76(79.2)$ & $19(26.8)$ & $64(70.3)$ & $19(28.8)$ & $57(62.0)$ \\
MADRS remitter, $\mathrm{n}(\%)$ & $27(43.5)$ & $7(7.3)$ & $36(50.7)$ & $17(18.7)$ & $34(51.5)$ & $16(17.4)$ \\
MADRS non-remitter, $\mathrm{n}(\%)$ & $35(56.5)$ & $89(92.7)$ & $35(49.3)$ & $74(81.3)$ & $32(48.5)$ & $76(82.6)$ \\
Total, $\mathrm{n}(\%)$ & $62(39.2)$ & $96(60.8)$ & $71(43.8)$ & $91(56.2)$ & $66(41.8)$ & $92(58.2)$ \\
\hline
\end{tabular}

Notes: Data are $\mathrm{n}(\%)$, where \% is a proportion of the larger total. Early improvement was defined as a reduction in MADRS total score of at least $20 \%$ from baseline to week 2. Patients who did not satisfy the criteria for early improvement were defined as non-early improvers. MADRS response was defined as at least a $50 \%$ decrease in MADRS total score from baseline, and remission was defined as a decrease in MADRS total score to 10 or lower.

Abbreviation: MADRS, Montgomery-Åsberg Depression Rating Scale.

Table 2 Sensitivity, Specificity, and Predictive Values of Early Improvement for Response and Remission at Week 8

\begin{tabular}{|c|c|c|c|c|}
\hline & Placebo $(n=158)$ & $\begin{array}{l}\text { Vortioxetine } 10 \mathrm{mg} \\
(n=162)\end{array}$ & $\begin{array}{l}\text { Vortioxetine } 20 \mathrm{mg} \\
(\mathrm{n}=\mid 58)\end{array}$ & $\begin{array}{l}\text { Vortioxetine } 10 \mathrm{mg} \text { or } \\
20 \mathrm{mg}(\mathrm{n}=320)\end{array}$ \\
\hline \multicolumn{5}{|l|}{ MADRS response } \\
\hline Sensitivity (\%) & 66.1 & 65.8 & 57.3 & 61.5 \\
\hline Specificity (\%) & 76.8 & 77.1 & 75.0 & 76.1 \\
\hline Positive predictive value (\%) & 62.9 & 73.2 & 71.2 & 72.3 \\
\hline Negative predictive value (\%) & 79.2 & 70.3 & 62.0 & 66.1 \\
\hline \multicolumn{5}{|l|}{ MADRS remission } \\
\hline Sensitivity (\%) & 79.4 & 67.9 & 68.0 & 68.0 \\
\hline Specificity (\%) & 71.8 & 67.9 & 70.4 & 69.1 \\
\hline Positive predictive value (\%) & 43.5 & 50.7 & 51.5 & 51.1 \\
\hline Negative predictive value (\%) & 92.7 & 81.3 & 82.6 & 82.0 \\
\hline
\end{tabular}

Note: MADRS response was defined as at least a 50\% decrease in MADRS total score from baseline, and remission was defined as a decrease in MADRS total score to 10 or lower.

Abbreviation: MADRS, Montgomery-Åsberg Depression Rating Scale.

MDD can make it difficult for physicians to determine the most suitable treatment regimen for each patient. Emerging evidence suggests that mood disorders are not discrete categories of specific diseases, but rather, form a continuous disease spectrum. ${ }^{18}$ Furthermore, genetic, neurobiological and environmental factors may influence the pathophysiology of MDD; ${ }^{19}$ as such, "effective" treatment can vary from patient to patient. Premature termination and repeated switching of medication may lead to treatment fatigue, prolongation of suffering, newly emerging adverse events, and escalating medical costs for patients. ${ }^{20-22}$ Early improvement as a predictor of future response may guide treatment strategy by encouraging physicians to continue with treatment regimens that have led to a partial initial response. The positive predictive values reported in this post hoc analysis indicate that future response to vortioxetine may be predicted by partial improvement from baseline in MADRS total score (at least $20 \%$ ) after 2 weeks of treatment. However, it should be noted that such changes in MDD severity may be difficult to distinguish in real-world practice with tools used in clinical trials. ${ }^{4}$ Development of simple, practice-friendly tools capable of identifying early partial improvement with antidepressants may be crucial for supporting clinical decision-making and encouraging potential responders to continue with treatment.

Positive predictive values of early improvement were relatively high for vortioxetine (response: $72.3 \%$; remission: $51.1 \%$ ) compared with those for antidepressants assessed in a previous systematic review, including selective serotonin reuptake inhibitors (SSRIs) and a selective serotonin-norepinephrine reuptake inhibitor (overall response: $63.0 \%$; overall remission: $33.0 \%$ ), although it should be noted that the methodology for the post hoc 
analysis varied from the studies reviewed. ${ }^{8}$ Antidepressants, even those with the same mechanism of action, can have variable positive predictive values: the positive predictive value of vortioxetine was similar to the SSRI fluoxetine but not paroxetine, for example. ${ }^{8}$ Negative predictive values were lower for vortioxetine (response: 66.1\%; remission: $82.0 \%$ ) than for other antidepressants overall (response: $77.0 \%$; remission: 90.0\%). Values for vortioxetine were again similar to those seen with some, but not all, SSRIs. ${ }^{8}$

These results could prove valuable to physicians when making treatment decisions. ${ }^{4,9}$ Moderate sensitivity and comparatively low negative predictive values of early improvement may indicate that patients with MDD who do not demonstrate improvements in MADRS score 2 weeks after treatment initiation could potentially still benefit from longer-term treatment with vortioxetine. Even if unfavorable outcomes can be predicted to some extent, physicians may lack confidence in terminating a medication regimen unless the negative predictive value of early improvement is $100 \%$. Although not statistically assessed, the lower negative predictive value for response seen with vortioxetine $20 \mathrm{mg}(62.0 \%)$ compared with vortioxetine $10 \mathrm{mg}(70.3 \%)$ may reflect a greater potential for response after 2 weeks with the higher dose of vortioxetine, even if early improvement is not observed.

There are some limitations to this study. First, the results were obtained from a post hoc analysis of clinical trial data; the examination of week 8 response and remission rates in subgroups stratified by early improvement of MADRS score were not prespecified. Second, all patients included in the post hoc analysis had recurrent MDD. Although response and remission rates with vortioxetine were similar to those seen in previous trials enrolling Japanese patients experiencing their first episode of MDD, ${ }^{13,23}$ we cannot rule out the possibility that disease characteristics may have influenced the response and remission rates in this study. Third, the predictability of future response and remission was examined only in a limited population of patients with MDD treated with vortioxetine, thus the results cannot be generalized to all patients with MDD or directly compared with those of other antidepressants.

\section{Conclusions}

In conclusion, future response with vortioxetine may be predicted by a partial improvement in MADRS score as early as 2 weeks after treatment initiation, but patients could still benefit from longer-term treatment with vortioxetine even in the absence of early improvement. These findings can support physicians and patients in making treatment decisions with respect to vortioxetine.

\section{Abbreviations}

DSM, Diagnostic and Statistical Manual of Mental Disorders; MADRS, Montgomery-Åsberg Depression Rating Scale; MDD, major depressive disorder; SSRI, selective serotonin reuptake inhibitor.

\section{Data Sharing Statement}

The data sets, including the redacted study protocol, redacted statistical analysis plan, and individual participant data supporting the results reported in this article, will be made available by the corresponding author within 3 months from initial request to researchers who provide a methodologically sound proposal. The data will be provided after its de-identification, in compliance with applicable privacy laws, data protection, and requirements for consent and anonymization.

\section{Acknowledgments}

We would like to pay our respects to Dr Yoshinori Watanabe (Himorogi Psychiatric Institute, Tokyo, Japan), who passed away in August of 2021, shortly before submission of this manuscript. We are grateful for his contributions as an investigator in the primary study and the development of this manuscript. Y.W. received speaker's honoraria from Janssen Pharmaceutical K.K., Meiji Seika Pharma Co., Ltd, Mitsubishi Tanabe Pharma Corp., Mochida Pharmaceutical Co., Ltd, Otsuka Pharmaceutical Co., Ltd, Pfizer Japan Inc., Sumitomo Dainippon Pharma Co., and Takeda Pharmaceutical Co., Ltd.

We are grateful to current and former employees of Takeda Pharmaceutical Co. Ltd and Lundbeck Japan for their contributions to the study: Akira Nishimura and Kiyofumi Sasai (executed the primary clinical trial); Takafumi Akimoto and Aya Makita (supported the design of the analysis); and Isao Inada, Akihiro Takano, and Manami Imazaki (supported the interpretation of the results). Medical writing support was provided by Svetha Sankar, BSc, BVMS, of Oxford PharmaGenesis, Melbourne, Australia and was funded by Takeda Pharmaceutical Co., Ltd.

\section{Author Contributions}

All authors made a significant contribution to the work reported, whether that is in study conception, study design, 
execution, acquisition of data, analysis and interpretation, or in all these areas; took part in drafting, revising, or critically reviewing the article; gave final approval of the version to be published; agreed on the journal to which the article has been submitted; and agree to be accountable for all aspects of the work.

\section{Funding}

This study was funded by Takeda Pharmaceutical Co., Ltd.

\section{Disclosure}

T.I. served as medical officer of the primary study; has received personal fees from Daiichi Sankyo, Janssen Pharmaceuticals, Lilly, Mochida Pharmaceutical Co., Ltd, MSD, Taisho Toyama Pharmaceutical, Takeda Pharmaceutical Co., Ltd, and Yoshitomiyakuhin; has received grants from Astellas, Eisai, Shionogi Inc., and Tsumura; has received grants and personal fees from Kyowa Pharmaceutical Industry, Meiji Seika Pharma Co., Ltd, Mitsubishi Tanabe Pharma Corp., Novartis Pharma, Otsuka Pharmaceutical Co., Ltd, Pfizer, and Sumitomo Dainippon Pharma; and is a member of the advisory boards of Mitsubishi Tanabe Pharma Corp., Novartis Pharma, and Pfizer. S.F., T.M., T.K., K.I., T.N., and K.F. are employees of Takeda Pharmaceutical Co., Ltd. Y.M. is an employee of Lundbeck Japan K.K. K.W. has received manuscript fees or speaker's honoraria from Astellas, Daiichi Sankyo, GlaxoSmithKline, Janssen Pharmaceuticals, Kyowa Pharmaceutical Industry, Lilly, Meiji Seika Pharma Co., Ltd, Mitsubishi Tanabe Pharma Corp., MSD, Otsuka Pharmaceutical Co., Pfizer, Shionogi Inc., Sumitomo Dainippon Pharma Co., Ltd, Takeda Pharmaceutical Co., Ltd, and Yoshitomi; has received research and grant support from Astellas, Daiichi Sankyo, Eisai, Meiji Seika Pharma Co., Ltd, Mitsubishi Tanabe Pharma Corp., MSD, Otsuka Pharmaceutical Co., Ltd, Pfizer, and Shionogi Inc.; is a consultant for Eisai, Janssen Pharmaceutical, Kyowa Pharmaceutical, Lilly, Mitsubishi Tanabe Pharma Corp., Otsuka Pharmaceutical Co., Ltd, Pfizer, Sumitomo Dainippon Pharma Co., Ltd, and Taisho Toyama Pharmaceutical; and is a consultant and advisory board member for Takeda Pharmaceutical Co. Ltd, Lundbeck Japan, and Viatris, outside the submitted work.

\section{References}

1. Paykel ES. Depression: major problem for public health. Epidemiol Psichiatr Soc. 2006;15:4-10. doi:10.1017/S1121189X00001974
2. Sado M, Yamauchi K, Kawakami N, et al. Cost of depression among adults in Japan in 2005. Psychiatry Clin Neurosci. 2011;65:442-450. doi:10.1111/j.1440-1819.2011.02237.x

3. Machado-Vieira R, Baumann J, Wheeler-Castillo C, et al. The timing of antidepressant effects: a comparison of diverse pharmacological and somatic treatments. Pharmaceuticals. 2010;3:19-41. doi:10.3390/ ph3010019

4. Oluboka OJ, Katzman MA, Habert J, et al. Functional recovery in major depressive disorder: providing early optimal treatment for the individual patient. Int J Neuropsychopharmacol. 2018;21:128-144. doi:10.1093/ijnp/pyx081

5. Nakajima S, Uchida H, Suzuki T, et al. Is switching antidepressants following early nonresponse more beneficial in acute-phase treatment of depression?: a randomized open-label trial. Prog Neuropsychopharmacol Biol Psychiatry. 2011;35:1983-1989. doi:10.1016/j.pnpbp.2011.08.008

6. Katz MM, Tekell JL, Bowden CL, et al. Onset and early behavioral effects of pharmacologically different antidepressants and placebo in depression. Neuropsychopharmacology. 2004;29:566-579. doi:10.1038/sj.npp.13 00341

7. Committee for Treatment Guidelines of Mood Disorders, Japanese Society of Mood Disorders. Guidelines for treatment of depression (DSM-5)/major depression disorder (in Japanese); 2016. Available from: https://www.secretariat.ne.jp/jsmd/iinkai/katsudou/data/ 160731.pdf. Accessed February 5, 2021.

8. Wagner S, Engel A, Engelmann J, et al. Early improvement as a resilience signal predicting later remission to antidepressant treatment in patients with major depressive disorder: systematic review and meta-analysis. J Psychiatr Res. 2017;94:96-106. doi:10.1016/j. jpsychires.2017.07.003

9. Szegedi A, Jansen WT, van Willigenburg AP, et al. Early improvement in the first 2 weeks as a predictor of treatment outcome in patients with major depressive disorder: a meta-analysis including 6562 patients. J Clin Psychiatry. 2009;70:344-353. doi:10.4088/ JCP. $07 \mathrm{~m} 03780$

10. Sanchez C, Asin KE, Artigas F. Vortioxetine, a novel antidepressant with multimodal activity: review of preclinical and clinical data. Pharmacol Ther. 2015;145:43-57. doi:10.1016/j.pharmthera.2014.07.001

11. Thase ME, Mahableshwarkar AR, Dragheim M, et al. A meta-analysis of randomized, placebo-controlled trials of vortioxetine for the treatment of major depressive disorder in adults. Eur Neuropsychopharmacol. 2016;26:979-993. doi:10.1016/j. euroneuro.2016.03.007

12. Inoue $T$, Sasai K, Kitagawa $T$, et al. Randomized, double-blind, placebo-controlled study to assess the efficacy and safety of vortioxetine in Japanese patients with major depressive disorder. Psychiatry Clin Neurosci. 2020;74(2):140-148. doi:10.1111/pcn.12956

13. Nishimura A, Aritomi Y, Sasai K, et al. Randomized, double-blind, placebo-controlled 8-week trial of the efficacy, safety, and tolerability of 5,10 , and $20 \mathrm{mg} /$ day vortioxetine in adults with major depressive disorder. Psychiatry Clin Neurosci. 2018;72:64-72. doi:10.1111/pen.12565

14. International Conference on Harmonisation of Technical Requirements for Registration of Pharmaceuticals for Human Use. ICH harmonized tripartite guideline: guideline for good clinical practice. J Postgrad Med. 2001;47:45-50.

15. American Psychiatric Association. Diagnostic and Statistical Manual of Mental Disorders. 5th ed. Arlington (VA): American Psychiatric Publishing; 2013.

16. World Health Organization. Recurrent depressive disorder - international statistical classification of diseases and related health problems 10th revision (ICD-10); 2019. Available from: https://icd.who.int/ browse10/2019/en\#F33. Accessed November 3, 2021.

17. Habert J, Katzman MA, Oluboka OJ, et al. Functional recovery in major depressive disorder: focus on early optimized treatment. Prim Care Companion CNS Disord. 2016;1:18. doi:10.4088/ PCC.15r01926 
18. Ng QX, Lim DY, Chee KT. Reimagining the spectrum of affective disorders. Bipolar Disord. 2020;22:638-639. doi:10.1111/bdi.12960

19. Mariani N, Cattane N, Pariante C, et al. Gene expression studies in Depression development and treatment: an overview of the underlying molecular mechanisms and biological processes to identify biomarkers. Transl Psychiatry. 2021;11:354. doi:10.1038/s41398021-01469-6

20. Cohen LJ, Sclar DA, Culpepper L, et al. Discussion: a fresh look at monoamine oxidase inhibitors for depression. $J$ Clin Psychiatry. 2012;73(Suppl 1):42-45. doi:10.4088/JCP.11096sulc.07

21. Al-Harbi KS. Treatment-resistant depression: therapeutic trends, challenges, and future directions. Patient Prefer Adherence. 2012;6:369-388. doi:10.2147/PPA.S29716
22. Leuchter AF, Cook IA, Hunter AM, et al. A new paradigm for the prediction of antidepressant treatment response. Dialogues Clin Neurosci. 2009;11:435-446. doi:10.31887/DCNS.2009.11.4/afleuchter

23. Inoue T, Nishimura A, Sasai K, et al. Randomized, 8-week, double-blind, placebo-controlled trial of vortioxetine in Japanese adults with major depressive disorder, followed by a 52-week open-label extension trial. Psychiatry Clin Neurosci. 2018;72:103-115. doi:10.1111/pcn.12623

\section{Publish your work in this journal}

Neuropsychiatric Disease and Treatment is an international, peerreviewed journal of clinical therapeutics and pharmacology focusing on concise rapid reporting of clinical or pre-clinical studies on a range of neuropsychiatric and neurological disorders. This journal is indexed on PubMed Central, the 'PsycINFO' database and CAS, and is the official journal of The International Neuropsychiatric Association (INA). The manuscript management system is completely online and includes a very quick and fair peer-review system, which is all easy to use. Visit http://www.dovepress.com/testimonials.php to read real quotes from published authors. 